\title{
CUADRILATERO VENOSO DE ROGIE: VARIANTES ANATOMICAS
}

\author{
Rogie's Venous Quadrilateral: Anatomical Variants
}

${ }^{*}$ Roberto Orlando Pineda Leiva, ${ }^{* *}$ Marvin Jesús Villanueva Márquez, **Gustavo Adolfo Rodríguez Rivera

\section{RESUMEN}

El cuadrilátero venoso de Rogie es una región anatómica en donde las venas mesentérica inferior, esplénica y mesentérica superior van a tributar en la vena porta hepática. La vena porta hepática es un vaso de gran calibre que recoge la sangre venosa del estómago, yeyuno, íleon, duodeno, colon y parte del sigmoide. En el laboratorio de anatomía macroscópica humana de la Universidad Nacional Autónoma de Honduras en el Valle de Sula (UNAHVS) encontramos dos variantes anatómicas de las estructuras que componen el cuadrilátero venoso de Rogie en relación a la aorta abdominal y en la manera en que tributa la vena mesentérica inferior. La primera variante que se encontró fue la vena mesentérica inferior drenando directamente al ángulo de unión entre la vena mesentérica superior y la vena esplénica. La segunda variante se encontró en la misma región del mismo cadáver, en el límite posteroinferior del cuadrilátero de Rogie, en donde la vena renal izquierda discurre posterior a la aorta abdominal. La posición retroaórtica de la vena renal izquierda puede resultar perjudicial para la persona en caso de ser comprimida por la aorta abdominal.

\section{PALABRAS CLAVE}

Aorta abdominal, Anatomía, Disección, Hematuria, Varicocele, Vena Porta.

\section{ABSTRACT}

The Rogie's venous quadrilateral is an anatomical region where the inferior mesenteric vein, splenic and superior mesenteric veins drain in the hepatic portal vein. The hepatic portal vein is a large caliber vessel that collects venous blood from the stomach, jejunum, ileum, duodenum, colon and part of the sigmoid. In the human macroscopic anatomy laboratory at Universidad Nacional Autónoma de Honduras en el Valle de
Sula (UNAH-VS) we found two anatomical variants the components of the Rogie's venous quadrilateral in relation to the abdominal aorta and the way the inferior mesenteric vein drains. The first variant found was the inferior mesenteric vein draining directly into junction between the splenic and superior mesenteric veins. The second variant found was in the same region and body in the post inferior limit of the Rogie's venous quadrilateral, where the left renal vein runs posterior to the abdominal aorta. This retro aortic position of the left renal vein could be harmful in case the abdominal aorta compressing it.

\section{KEY WORDS}

Abdominal Aorta, Anatomy, Disection, Hematuria, Portal Vein, Varicocele.

\section{INTRODUCCIÓN}

El cuadrilátero venoso de Rogie representa la unión de distintas venas y se encuentra posterior al cuello del páncreas, este cuadrilátero está limitado de la siguiente manera; medialmente la vena mesentérica superior, lateralmente la vena mesentérica inferior, superiormente la vena esplénica y posteroinferiormente la vena renal izquierda. Ver figura No. 1

Figura $\mathbf{N}^{\circ}$ 1: Esquema del Cuadrilátero Venoso de Rogie

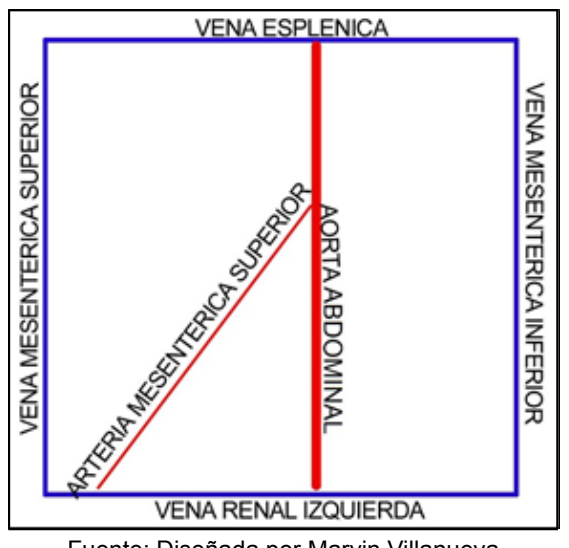

Fuente: Diseñada por Marvin Villanueva

*Jefe del Departamento de Ciencias Básicas de la Salud.

Escuela Universitarias de las Ciencias de la Salud. (EUCS, UNAH-VS)

**Estudiantes III año, Carrera de Medicina y Cirugía. EUCS, UNAH-VS

Dirigir correspondencia a: mjvmvm630@gmail.com

Recibido: 24 de marzo del 2,017 Aprobado: 01 de mayo 2017 
La unión de las venas esplénica, mesentérica inferior y mesentérica superior forma la vena porta hepática, que es una vena de gran calibre que recoge la sangre venosa del estómago, yeyuno, íleon, duodeno, colon y parte del sigmoide.

- La vena mesentérica superior drena la sangre del intestino delgado, ciego, yeyuno, la mitad derecha del colon transverso y colon ascendente. El origen de su tronco principal se encuentra posterior a la porción horizontal del duodeno y asciende verticalmente por detrás de la cabeza del páncreas para ir a unirse a la vena porta hepática. ${ }^{(1,2)}$

- La vena mesentérica inferior drena la sangre del recto, colon descendente, colon sigmoideo, la mitad izquierda del colon transverso y la flexura esplénica, comienza como la vena rectal superior y asciende, recibiendo tributarias de la vena sigmoidea, las tres venas cólicas y las venas hemorroidales, y termina uniéndose a la vena esplénica. ${ }^{(3)}$

- La vena esplénica drena sangre principalmente del bazo y la curvatura mayor del estómago; tiene su origen a partir de tres ramas venosas en el hilio esplénico e inicialmente recorre oblicuamente hacia abajo para encontrarse con la cola del páncreas. Por lo tanto, la vena recorre el borde superior de la cara anterior y posterior del páncreas para eventualmente unirse a la vena mesentérica superior. ${ }^{(1,2)}$

Las venas renales son de longitud larga y usualmente se encuentran anterior a la arteria renal en el hilio renal. La vena renal izquierda es casi tres veces más larga que la derecha, surge del segmento renal de la vena cava inferior formado a partir de la anastomosis subsupracardinal, una red venosa situada circunferencialmente alrededor de la aorta (collar renal). Eventualmente, el segmento posterior del collar desiste y la parte anterior persiste dando lugar a la vena renal izquierda. ${ }^{(4)} \mathrm{La}$ vena renal izquierda tiene una longitud de 6 a $10 \mathrm{~cm}$ y desemboca en la cara lateral izquierda de la vena cava inferior después de pasar por detrás de la arteria mesentérica superior y por delante de la aorta. Mientras que la vena renal derecha generalmente tiene una longitud de 2 a $4 \mathrm{~cm}$ y desemboca en la vena cava inferior por la cara derecha o por la posterolateral. La vena renal izquierda en comparación con la vena renal derecha, desemboca en la vena cava inferior en un nivel ligeramente más alto y en una localización más anterolateral. Además, la vena renal izquierda recibe a la vena supra- rrenal izquierda por arriba, la vena lumbar por detrás y la vena gonadal izquierda por abajo. En general la vena renal derecha no recibe ninguna rama. ${ }^{(1,5,6)}$

El objetivo de esta publicación, es dar a conocer el hallazgo de dos variantes anatómicas en un mismo cadáver.

\section{CASO CLINICO}

Se presentan los hallazgos encontrados en un cadáver masculino de entre 45 y 50 años de edad aproximadamente, indigente y por lo tanto, se desconoce si presentaba manifestaciones clínicas. Fue preparado según las normas del Manual de Disección de la Cátedra de Anatomía Macroscópica de la UNAH-VS con una solución de formaldehido al $35 \%$, alcohol al $95 \%$, glicerina, cloruro de sodio, tritón. Se aborda el retro peritoneo a través de la transcavidad de los epiplones dividiendo el ligamento gastrocólico y exponiendo el páncreas, procedemos a la disección y dividiendo la cabeza y el cuerpo del páncreas a través de su istmo para exponer el cuadrilátero venoso de Rogie. En la disección se encontraron dos variantes anatómicas de los componentes del cuadrilátero venoso de Rogie, en relación a la aorta y al sitio dónde tributaba la vena mesentérica inferior. Ver figura No. 2.

1. La primera variante encontrada fue que la vena mesentérica inferior tributaba al ángulo de unión entre la vena mesentérica superior y la vena esplénica.

2. La segunda variante se encontró también en el mismo cadáver, en el límite posteroinferior del cuadrilátero de Rogie, en donde la vena renal izquierda estaba retroaórtica. De acuerdo a la disposición anatómica común y las variantes anatómicas encontradas, se logró hacer el siguiente esquema comparativo. 
Figura $\mathbf{N}^{\circ}$ 2: Esquema comparativo del Cuadrilátero Venoso de Rogie.

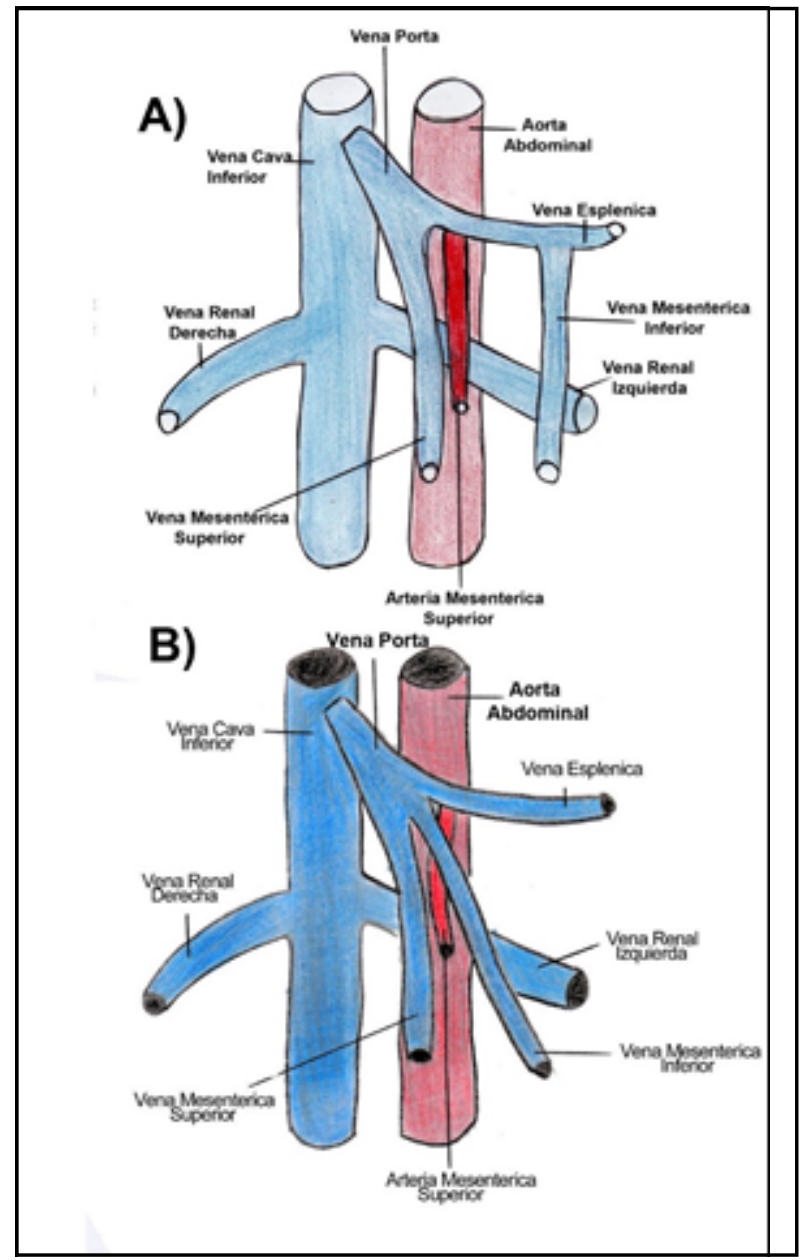

Obsérvese: A) Disposición normal de la vena mesentérica inferior y la vena renal en el cuadrilátero de Rogie. B) Variante anatómica, la vena mesentérica inferior va directo al ángulo de unión y la vena renal izquierda es retroaórtica.

Fuente: diseñada por Marvin Villanueva

Las variantes anatómicas son observables en el cadaver, (Ver figura No. 3) en el cual la vena mesentérica inferior va a tributar directamente al ángulo de unión entre las dos venas, la esplénica y la mesentérica superior y también se observa la vena renal izquierda posterior a la aorta.
Figura $\mathbf{N}^{`} 3$ : Foto del cadáver, con las variantes anatómicas.

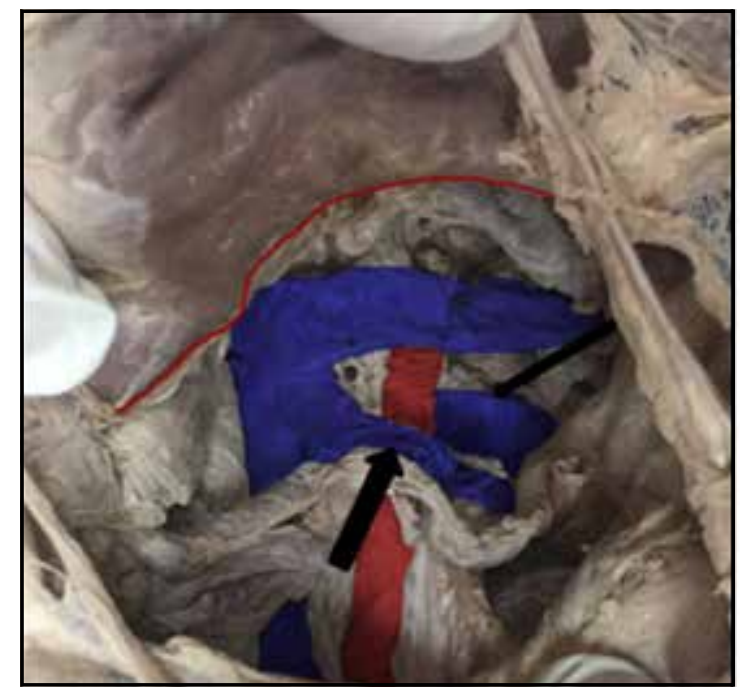

La flecha gruesa, señala la vena mesentérica inferior y la flecha delgada, señala la vena renal izquierda.

Fuente: Cadáver del caso

\section{DISCUSIÓN}

Al revisar la literatura anatómica internacional y comparar los textos oficiales de anatomía humana macroscópica, la frecuencia con que la vena mesentérica inferior tributa en el ángulo de unión entre la vena esplénica y la vena mesentérica superior es del $32 \%$, en la vena esplenica $38 \%$ y en la vena mesentérica superior $30 \%$. La vena renal izquierda retroaórtica es una variante anatómica poco común que usualmente tributa en la vena cava inferior un poco más abajo que la vena renal derecha. ${ }^{(7)}$ La frecuencia con la que la vena renal izquierda es retroaórtica es del $2 \% .{ }^{(8-10)}$ En el laboratorio de anatomía macroscópica humana de UNAH-VS encontramos las dos variantes anatómicas en el mismo cadáver.

Las anomalías de las venas renales son menos comunes que las de las arterias renales. La vena renal izquierda retroaórtica resulta de la persistencia de la anastomosis intersupracardinal y de la porción dorsal de la anastomosis subsupracardinal junto con la degeneración de la anastomosis intersubcardinal y la porción ventral de la anastomosis subsupracardinal. (11) Ver figura No. 4 
Figura No. 4. Desarrollo embriológico de la vena renal.

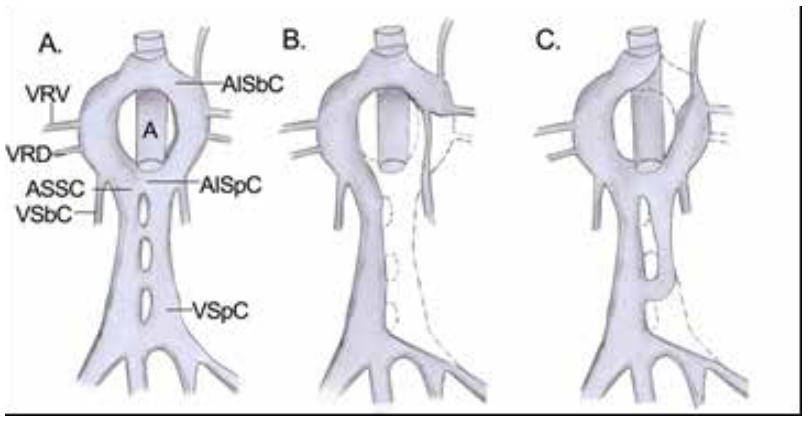

Obsérvese: A) Disposición de las venas del collar renal en embrión de 7 semanas. B) Desarrollo normal de las venas renales. C) Desarrollo anormal de las venas renales, vena renal izquierda retroaórtica. A, Aorta. VRV, Vena renal ventral. VRD, Vena renal dorsal. ASSC, Anastomosis subsupracardinal. VSbC, Vena subcardinal. VSpC, Vena supracardinal. AISpc, anastomosis intersupracardinal. AISbC, anastomosis intersubcardinal. Fuente: Diseñada por Marvin Villanueva

Las venas renales izquierdas retroaórticas pueden ser de dos tipos, la vena renal izquierda retroaórtica tipo I, pasa directamente por debajo de la aorta y va a drenar a la vena cava inferior en su sitio normal, a nivel de L2. La vena renal izquierda retroaórtica tipo II, cruza por debajo de la aorta y va a drenar a la vena cava inferior a nivel de L4/L5. ${ }^{(12,13)}$ El cadáver en el que encontramos dicha variante anatómica presentaba la vena renal izquierda retroaórtica tipo I.

Esta variante puede algunas veces causar síntomas clínicos, puede ser comprimida por la aorta, dando como resultado en un "síndrome de Nutcracker" con hipertensión, hematuria, proteinuria ortostática, dolor en flanco izquierdo. ${ }^{(14,15)}$ Asociado también al desarrollo de varicocele en hombres, trombosis de vena renal postparto en mujeres, pierna izquierda varicosa, síndrome de congestión pélvica y varices en la vulva. ${ }^{(11,16)}$ El tratamiento incluye la trasposición de la vena renal izquierda, el autotransplante renal, la nefropexia, nefrectomía laparoscópica y la colocación de stents. ${ }^{(16)}$ Estas anomalías pueden encontrarse mediante una tomografía axial computarizada (TAC), angiorresonancia magnética, ecografía Doppler y ultrasonido. EI TAC y la angiorresonancia magnética por su elevada sensibilidad $(>95 \%)$ y especificidad $(>90 \%)$ son las técnicas más precisas y utilizadas. La ecografía Doppler puede alcanzar una sensibilidad de $85 \%$ y una especificad de $92 \%$ y puede revelar la velocidad del flujo sanguíneo en la vena renal. El ultrasonido no es muy exacto. (17) Al paciente no se le realizaron ninguno de estos exámenes puesto que se trataba de un indigente en el cual se desconocían sus condiciones y su historial clínico. Se pudieron descubrir las dos varian- tes anatómicas en la disección del mismo durante una catedra de Anatomía Macroscópica Humana.

Se consultó otro caso clínico en el cual se examinaron a nueve pacientes en el hospital de la Universidad Nacional de Pusan en Yangsan, República de Corea entre el año 2003 y 2009; Ocho resultaron con vena renal izquierda retroaórtica y uno con vena renal izquierda circumaortica, cinco de ellos eran hombres y cuatro mujeres, los síntomas urológicos del diagnóstico inicial fueron variados, microhematuria en cinco de los nueve pacientes, dolor en flanco izquierdo en cuatro de los nueve pacientes, dolor inguinal en uno de los cinco pacientes masculinos, hematuria macroscópica en uno de los nueve pacientes, varicocele en dos de los cinco pacientes masculinos, seis pacientes presentaron vena renal izquierda retroaórtica tipo I, y dos pacientes presentaron vena renal izquierda retroaórtica tipo II, uno de los pacientes se sometió a una nefrectomía laparoscópica debido a un riñón no funcional con varicocelectomía. Los sintomas de los cinco pacientes con hematuria microscópica continuó, mientras que el paciente con hematuria macroscópica mejoro después de la nefrectomía. ${ }^{(18)}$

\section{Conclusión:}

Hacer un buen interrogatorio y exploración física a todos los pacientes para saber si los síntomas que presenta están asociados a una vena renal izquierda retroaórtica y luego proseguir a realizar los estudios por imagen (radiológicos) pertinentes. Al momento de realizar una cirugía renal, se debe de realizar una buena planificación quirúrgica y tener muy presente esta variante anatómica. Si estas anomalías no se reconocen pueden causar daños renales severos y hemorragia.

Insistir en hacer obligatoria la autopsia el cual es un importante y necesario procedimiento para que la medicina siga avanzando, ya que así se obtiene más conocimiento sobre las causas de defunción. En el caso de las variantes anatómicas se puede brindar consejería a los familiares de quien la presento debido a que pueden desarrollarse por causa hereditaria.

\section{AGRADECIMIENTOS}

Al todo el personal de laboratorio de Anatomía de la Escuela Universitaria de las Ciencias de la Salud de la Universidad Nacional Autónoma de Honduras en el Valle de Sula. EUCS/ UNAH- VS. 


\section{REFERENCIAS BIBLIOGRAFICAS}

1. Gray H. Gray's Anatomy. New York: Barnes \& Noble; 2010.

2. Catalano C, Broglia L, Laghi A, Pediconi F, Napoli A. Magnetic Resonance imaging of the portal system In: Rossi P, Ricci P. Portal hypertension: diagnostic imaging and imaging-guided therapy. New York: Springer; 2000. p115-124.

3. Drake R, Vogl W, Mitchell A. Gray's anatomy for students. s.I: Elsevier Health Sciences; 2004.

4. Mozes G, Gloviczki P. Venous embriology and y anatomy. In: Bergan J, Bunke N. The Vein Book. USA: Oxford University Press; 2014. p 17-26.

5. Quaia E. Radiological imaging of the kidney. New York: Springer-Verlag; 2011.

6. Wein A, Kavoussi L. Campbell-Walsh Urology. 9 edth. USA: Elsevier; 2006.

7. Mandell J. Core radiology: a visual approach to diagnostic imaging. New York: Cambridge University Press; 2013.

8. Netter F. The Netter Collection of Medical Illustrations: digestive syste. Vol 9 2nd ed. China: Elsevier; 2017.

9. Tubaikh JA, Reiser MF. Congenital diseases and syndromes. New York: Springer; 2009.

10. Tublin M. Diagnostic imaging: genitourinary. 3 . s.l: Amirsys; 2015.

11. Mulliken J, Burrows P, Fishman S. Vascular anomalies: hemangiomas and malformations. New York: Oxford University Press; 2013.

12. Upchurch G, Criado E. Aortic aneurysms: pathogenesis and treatment. s.l: Humana Press; 2009.
13. Branchereau $A$, Jacobs $M$. Unexpected challenges in vascular surgery. USA: Blackwell Publishing; 2005.

14. Saba L, Suri J. Multi-detector CT imaging: abdomen, pelvis, and CAD applications. Boca Raton: CRC Press; 2013.

15. Saba L. Imaging of the cardiovascular system, thorax, and abdomen. Boca Raton: CRC Press; 2016.

16. Stevenson R, Hall J. Human malformations and related anomalies. 2nd edth. New York: Oxford University Press; 2006.

17. Payeras A, Lara P, Redon J. Manejo del Paciente Hipertenso en la Práctica Clínica. Madris: Médica Panamerica; 2009.

18. Nam JK, Park SW, Lee SD, Chung MK. The clinical significance of a retroaortic left renal vein. Korean J Urol. 2010;51(4):276-80. 